\title{
The Effects of Sap of Nitraria Plant on the Growth of Its Endogenous Nitrogen-fixing Microbes under Saline-Alkaline Stress
}

\author{
Jianfeng Li \\ Institute of Soil and Environment Bioremediation in \\ Karst habitats \\ Guizhou Normal College \\ Guiyang, China \\ e-mail:alexclover@foxmail.com \\ Shuqin Zhang \\ Key Laboratory for Grassland Ecosystem of Ministry of \\ Education \\ Gansu Agricultural University \\ Lanzhou, China \\ e-mail:smartclover@foxmail.com
}

\author{
Jianxiong $\mathrm{Du}^{*}$ \\ Guizhou University of Finance and Economics line 1 \\ Guiyang, China \\ e-mail: djx7401@yahoo.com.cn
}

\begin{abstract}
The endophytic N-fixing microbes Klebsiella oxytoca NHglj1, Klebsiella oxytoca NHglj2 and Enterobacter asburiae LMw107 of Nitraria were selected to explore the effect of Nitraria schoberi L. plant sap on salt and alkali resistance of its 3 endogenous $\mathrm{N}$-fixing microbes strains in the Winogradsky's $\mathrm{N}$-free medium under different salinity and alkalinity gradient. The results show that the $\mathrm{NaCl}$ tolerance of 3 strains is $0 \sim 4 \%$ and 3 strains can all survive in $\mathbf{p H ~} 6 \sim 13$. After sap of Nitraria is added, the salt resistance of NHglj1 decrease, the colony diameter of $K$. oxytoca NHglj1 is $59 \% \sim 82 \%$ that of control, the change of alkali resistance of $\mathrm{NHglj1}$ is similar to that of the salt resistance, the diameter of $\mathrm{NHglj} 1$ is $13 \% \sim 58 \%$ that of control $(P<0.05)$. However, the salt resistance of $\mathrm{NHglj2}$ and LMw107 increase, the diameter is larger than $2 \% \sim 45 \%$ that of the control. The alkali resistance of $\mathrm{NHglj2}$ and LMw107 also increase in pH6 13. The results exhibit that Nitraria sap may partially improve salt-alkali resistance of parts of endogenous $\mathbf{N}$-fixing microbes.
\end{abstract}

Keywords- Nitraria; Plant sap; Endogenous $N$-fixing microbes; Saline stress; $\mathrm{pH}$

\section{INTRODUCTION}

Endogenous $\mathrm{N}$-fixing microbes can utilize excess energy to fix nitrogen during the process of host plant tissue conduction and colonization, and macroscopically be regulated by plant regulating system [1], exhibits high $\mathrm{N}$-fixation efficiency and promote host growth by secreting auxin, phosphate solubilizing, enhancing plant resistance and so on stress resistance mechanisms[2]. Endogenous N-fixing microbes have enormous potentiality to supply nitrogen and enhance environmental adaptability, especially under situation of harsh climate and lack of nitrogen [3], so it displays a good application prospect in ecological restoration [4-5]. Symbiotic relationship between endogenous $\mathrm{N}$-fixing microbes and plant has been one of hot content of common concern in botany, microbiology, and ecology [6]. Environmental condition has an effect on invasion and colonization, $\mathrm{N}$ fixation and promoting plant growth, enhancing host antiadversity efficiency full play of endogenous $\mathrm{N}$-fixing microbes [7-8], the key to playing the positive role of endogenous $\mathrm{N}$-fixing microbes is if endogenous $\mathrm{N}$-fixing microbes can effectively colonize and establish stability and harmony combination [9]. So there is an important significance of theory and practice to study symbiosis nitrogen fixation mechanism between plant and endogenous $\mathrm{N}$-fixing microbes to understand adaptability of plant and microorganism, stress resistance, species diversity, and the role of ecological restoration under atrocious environment [10].

Nitraria schoberi. L is super-xerophytes, distributed in province of Inner Mongolia, Gansu, Xinjiang of China, and possesses the capabilities of drought tolerance, salinealkaline, sand burial, and resistance to wind erosion [11]. Sand burial branches of N. schoberi can grew out new adventitious root and accumulate sand into dune in damp sand, and form fixed, semi-fixed shrubs, so it has very important ecological status in arid and semiarid areas. Nie, et al. reported that Nitraria get nitrogen by endogenous $\mathrm{N}$-fixing microbes [12]. Herridge et al. confirm by $15 \mathrm{~N}$ dilution method that some sugarcane obtained $60 \%$ nitrogen of total nitrogen content through endogenous $\mathrm{N}$ fixing microbes [13].

The studies on Nitraria mainly involve vegetation succession, environmental adaptation, shifting sand fixation of its plant, in this research, we use the sap of Nitraria and endogenous $\mathrm{N}$-fixing microbes as materials to explore the effects of plant sap on saline-alkali tolerance of plant endogenous $\mathrm{N}$-fixing microbes, which reveal adaptability of Nitraria endogenous N-fixing microbes to rigor environment factor and the protection of Nitraria on strains. This research provides the materials and reference for exploring the symbiotic relationship 
between Nitraria endogenous N-fixing microbes and host, and biological nitrogen fixation under desert environment.

\section{MATERIALS AND METHODS}

\section{A. Materials and Medium}

Plant Materials and Habitat Survey of Plant Materials:

Minqin county of Gansu Province of China is one of typical oasises in desert, and bounded by Tenggeli desert and Badanjilin desert from three planes, sampling place belongs to temperate desert climate, and the annual evaporation is $2604.3 \mathrm{~mm}$. The majority landform type includes shrub coppice dunes and moving sand dunes [11].

Collection of Plant Materials: Wild Nitraria schoberi plants were collected at Xishawo area of Minqin county Gansu Province of China in August 2012, four semi-fixed sand dune plot with growing single community were randomly selected, five complete plants were collected at each plot. Fresh tissue samples and sandy soil were placed in chamber, and the original drying conditions were maintained as possible.

The Strains Tested: The strains indentified as Klebsiella oxytoca NHglj1, Klebsiella oxytoca. NHglj2, and Enterobacter asburiae LMw107 are separated and purified from root, stone, flesh of wild Nitraria separately.

Culture Media: Preparation of Winogradsky's N-free medium referred to Hara's method, $\mathrm{N}$-free plates with $1.6 \%$ agar [14].

\section{B. Test Method}

The preparation of the diluents of Nitraria leaves: The fresh and healthy leaves of Nitraria were washed 3-4 times with distilled water, after surface moisture was sipped up with filter paper, $1.0 \mathrm{~g}$ leaf was accurately weighed by electronic balance, The fresh leaf was grinded with being added $10 \mathrm{ml}$ sterile distilled water in aseptic technique platform, after washing mortar with sterile water, leaf lapping liquid of Nitraria was filtered 2 times with 0.45 um sterilized filter membrane, total $200 \mathrm{ml}$ sterile water was added in the whole process. Finally, diluents of surface sterile leaf were stored in refrigerator at $4^{\circ} \mathrm{C}$.

Culture Medium: Basic culture with being cooled to about $40^{\circ} \mathrm{C}$ was filled into Erlenmeyer flask (150ml), $49 \mathrm{ml}$ per bottle, according to the following test scheme to treatment setting.

a. Salt Resistance Test: salt concentration of basic culture was set at $0 \%, 1 \%, 2 \%, 3 \%, 4 \%$ and $5 \%$ through being added with $\mathrm{NaCl}$.

b. Alkali Resistance Test: $\mathrm{pH}$ of basic culture was set at 5, 6, 7, 8, 9, 10,11, 12 and 13, through being added with $\mathrm{HCl}, \mathrm{NaOH}$.

All above different gradient culture were sterilized at $121^{\circ} \mathrm{C}$ by moist heat in $26 \mathrm{~min}$, after culture was taken out, leaf lapping liquid of Nitraria was added into each gradient culture medium and sterile saline of the same salt concentration and alkali concentration were served as control, at last they were marked as "Y" group with being added into sap of Nitraria and "N" control group separately.

Inoculated and Culture: In aseptic operation platform, $1 \mathrm{ml}$ sterile sap of Nitraria was separately added into all Y group gradient $150 \mathrm{ml}$ Erlenmeyer flask with containing 49ml nitrogen-free culture and was shaken up, In addition $1 \mathrm{ml}$ sterile water was separately added into all "N" group, Erlenmeyer flask $(150 \mathrm{ml})$ with containing $49 \mathrm{ml} \mathrm{N}$-free culture $\left(50^{\circ} \mathrm{C}\right)$ and served as controls. $50 \mathrm{ml}$ culture which was placed in Erlenmeyer flask $(150 \mathrm{ml})$ was in poured into $9 \mathrm{~cm}$ Petri-dish. After culture was cooled solidification, three strains were streaked in $\mathrm{N}$-free plates in all treatment, 4 replications per strain. Plates were cultured at $27^{\circ} \mathrm{C}$ in a incubator at least $168 \mathrm{~h}$ until the colony diameter was measured.

\section{Data Procession and Analysis}

The data was presented as means \pm SE and differences of variables between treatments were compared using one way ANOVA followed by Dennett's test. The statistical software SPSS 16.0 was used.

\section{RESULTS AND ANALYSIS}

A. The effects of sap of Nitraria on the colony diameter of endogenous $\mathrm{N}$-fixing microbes under saline stress conditions

Fig .1 shows that the diameter of $K$. oxytoca NHglj1 exhibit significant differences $(P<0.05$ for all), after NHglj1 separated from the root of Nitraria is cultured for $168 \mathrm{~h}$ under different treatment. Under culture conditions with being added leaf lapping liquid of Nitraria (followings replaced by "Y" group) and control group (equivalent sterile water replace leaf lapping diluents, followings replaced by " $\mathrm{N}$ " group), the $\mathrm{NaCl}$ tolerance range of NHglj1 was 0 4\%, but NHglj1 can not survive under $5 \% \mathrm{NaCl}$. The results shows that the colony diameter decrease gradually with the increase of $\mathrm{NaCl}$ concentration under without being added sap of Nitraria, after being added sap of Nitraria, diameter increase firstly and then decrease gradually with the increase of $\mathrm{NaCl}$ concentration, and the diameter reaches the maximum value $(3.11 \sim 3.00 \mathrm{~mm})$ in $1 \sim 2 \% \mathrm{NaCl}$. The diameter of $\mathrm{Y}$ group is respectively $18 \%, 40 \%, 41 \%, 38 \%$ and $31 \%$ of $\mathrm{N}$ group, The results exhibit that sap of Nitraria can significantly decrease salt resistance of NHglj1 of $\operatorname{root}(P<0.05)$

Fig .2 shows that the diameter of K. oxytoca NHglj2 exhibit significant differences $(P<0.05)$, after NHglj2 separated from the stone of Nitraria is cultured for $168 \mathrm{~h}$ under different $\mathrm{NaCl}$ treatment. No matter with or without sap of Nitraria, the diameter of NHglj2 decrease with the increase of $\mathrm{NaCl}$ concentration in $0 \sim 5 \%$, but $\mathrm{NHglj} 2$ can not grow under $5 \% \mathrm{NaCl}$. After being added sap of Nitraria, the diameter of $\mathrm{Y}$ group is respectively 1.04 , $1.30,1.18,1.45$, and 1.36 times of $\mathrm{N}$ group in $0 \sim 4 \% \mathrm{NaCl}$, the changes show significant differences $(P<0.05)$ in $1 \sim 4 \% \mathrm{NaCl}$, the results suggest that sap of Nitraria can significantly increase salt resistance of NHglj2 of stone $(P<0.05)$

Fig .3 shows that the diameter of E. asburiae LMw107 exhibite significant differences $(P<0.05)$, after LMw107 separated from the stone of Nitraria is cultured for $168 \mathrm{~h}$ in solid culture under different $\mathrm{NaCl}$ concentration treatment. LMw107 is similar to NHglj2, no matter with or without sap of Nitraria, the diameter of LMw107 decrease with the increase of $\mathrm{NaCl}$ concentration in $0 \sim 5 \%$ $\mathrm{NaCl}$, after being added sap of Nitraria, the diameter of 
"Y” group is respectively 1.12, 1.05, 1.36 and 1.02 times of " $\mathrm{N}$ " group in $0 \sim 3 \% \mathrm{NaCl}, 86 \%$ of $\mathrm{N}$ group under $4 \%$ $\mathrm{NaCl}$, the changes in the diameter show significant differences $(P<0.05)$ in $0 \sim 4 \% \mathrm{NaCl}$.

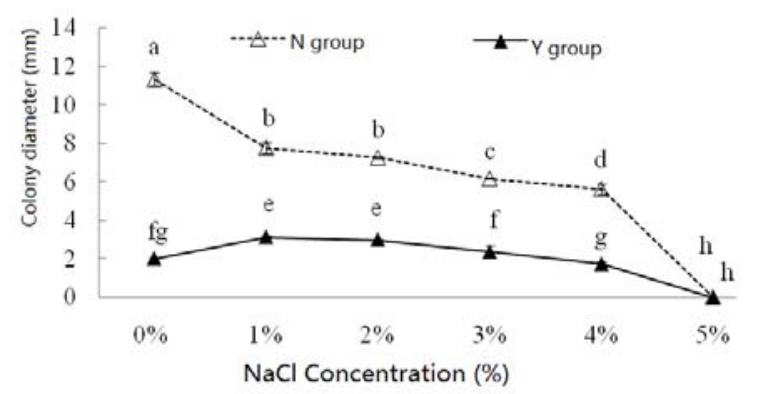

Figure 1. The effects of sap of Nitraria on the colony diameter of $K$. oxytoca NHglj1

* Note: the different small letters mean significant difference among the different treatments $(P<0.05)$, results are means \pm SD $(n=4)$. same blew.

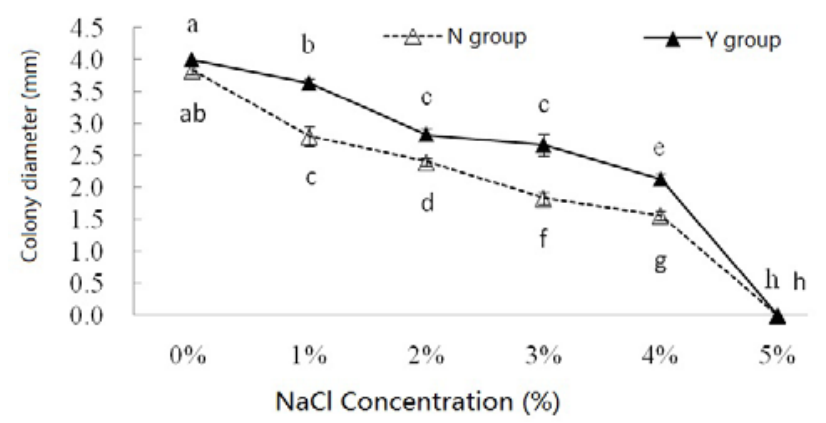

Figure 2. The effects of sap of Nitraria on the colony diameter of $K$. oxytoca NHglj2

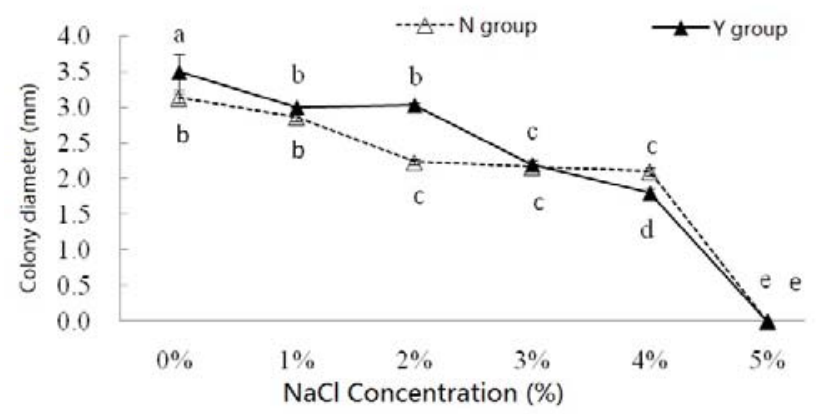

Figure 3. The effects of sap of Nitraria on the colony diameter of $E$. asburiae LMw107

B. The effects of sap of Nitraria on the colony diameter of endogenous $N$-fixing microbes under alkaline stress

Fig .4 shows that the diameter of K. oxytoca NHglj1 exhibite significant differences $(P<0.05)$, after NHglj1 separated from the root of Nitraria is cultured in solid culture medium for $168 \mathrm{~h}$ under different $\mathrm{pH}$ treatment. The diameter of $\mathrm{Y}$ and $\mathrm{N}$ group reach the maximum value in pH12. After being added sap of Nitraria, the diameter of Y group was respectively 35\%, 34\%, 35\%, 35\%, 34\%, $58 \%, 51 \%, 13 \%$ of $\mathrm{N}$ group in $\mathrm{pH} 6 \sim 13 \%$, the changes in the diameter show significant differences $(P<0.05)$. The results suggest that sap of Nitraria can significantly decrease acid and alkali resistance of NHglj1 $(P<0.05)$.

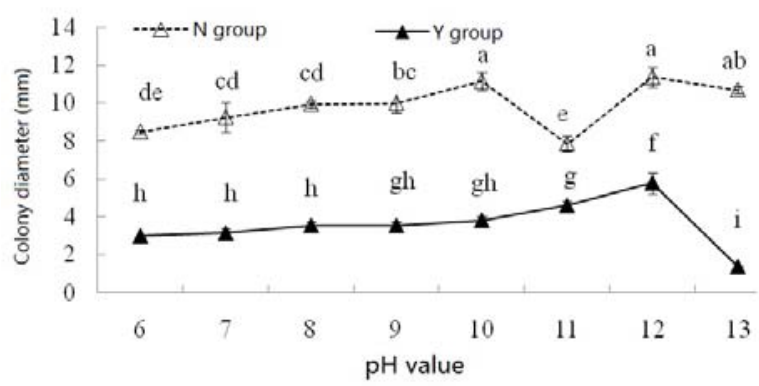

Figure 4. The effects of leaf diluents of Nitraria on the colony diameter of K. oxytoca NHglj1 under alkaline stress

Fig .5 shows that the diameter of $K$. oxytoca NHglj2 exhibit significant differences $(P<0.05)$ in solid culture for $168 \mathrm{~h}$ under different $\mathrm{pH}$. Without the sap of Nitraria, the diameter of $K$. oxytocaNHglj2 reach the maximum value in $\mathrm{pH}$ 8; Maximum diameter performs in $\mathrm{pH} 7$ under being added sap of Nitraria. The diameters of "Y" group are higher than those of " $\mathrm{N}$ " group in $\mathrm{pH} 6 \sim 13(P<0.05)$. The results indicate that sap of Nitraria can significantly increase alkali resistance of NHglj2 $(P<0.05)$ and improve colony forming speed of strains.

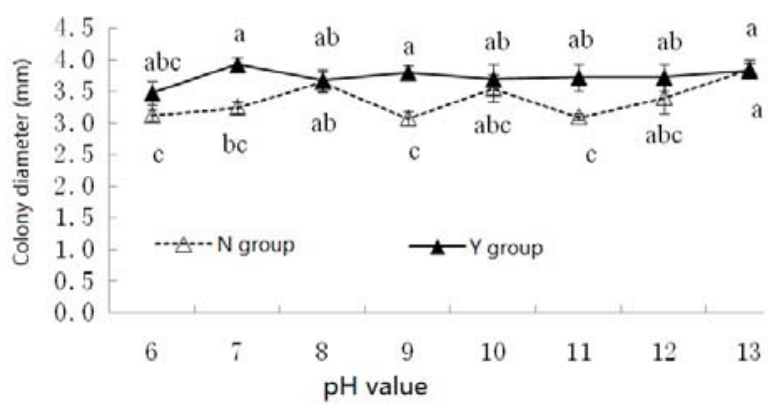

Figure 5. The effects of leaf diluents of Nitraria plant on the colony diameter of NHglj2 under alkaline stress

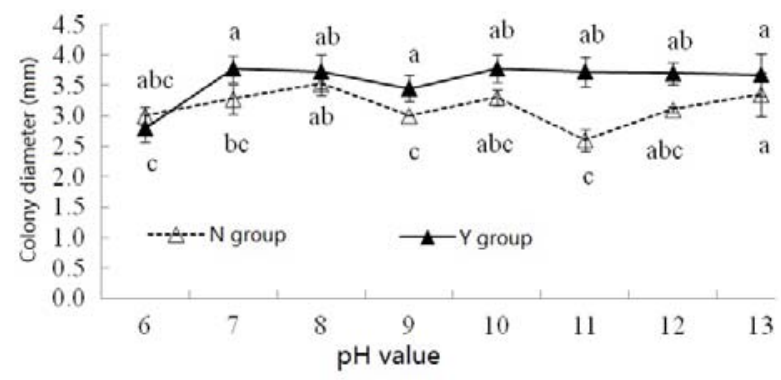

Figure 6. Effects of leaf sap of Nitraria plant on the colony diameter of E. asburiae LMw107 under alkaline stress

Fig .6 shows that the diameter of E. asburiae LMw107 exhibite significant differences $(P<0.05)$, after LMw107 separated from the flesh of Nitraria is cultured in solid culture for $168 \mathrm{~h}$ under different $\mathrm{pH}$ treatment. Under no sap of Nitraria, the diameter of LMw107 reach the 
maximum value $(3.53 \mathrm{~mm})$ in $\mathrm{pH}$, maximum value (3.78mm) in $\mathrm{pH} 7$ under being added sap of Nitraria. The diameters of $\mathrm{Y}$ group are higher than those of $\mathrm{N}$ group (respectively $P<0.05$ ) in $\mathrm{pH} 7 \sim 13$. This result is similar to that of NHglj2, which exhibit that sap of Nitraria can significantly increase alkali resistance of LMw107 $(P<0.05)$.

\section{DISCUSSION}

In this research, results show that $0.1 \mathrm{~g}$ fresh leaf sap per liter leaf sap performs the best on improve colony growth. In order to guarantee plant tissue grinding fluid to sustain action in the growth period of $\mathrm{N}$-fixing microbes, plant sap is immediately added into $\mathrm{N}$-free medium before it solidifies, instead of smearing plant sap on the surface of the solidified medium, but the operation is been on, due to the temperature of medium comes to solidified is at about $50^{\circ} \mathrm{C}$, which may be changed the composition or activities of some enzymes in plant sap.

The results show that the $\mathrm{NaCl}$ tolerance of 3 strains was $0 \sim 4 \%$, 3 strains could not grow at $5 \% \mathrm{NaCl}$, which shows that alkali resistance of 3 strains was extremely strong.

Zhang report that the sap of alfalfa plant reduced more auxin, exogenous Rhizobium polysaccharide from Sinorhizobium meliloti 12531f, Rhizobium meliloti GNf and Rhizobium meliloti GN5, and also promote the growth and proliferation of rhizobia [15]. That uniform with manifestation of $K$. oxytoca NHglj2 and LMw107 in this research, but manifestation of NHglj1 is just contrary.

\section{CONCLUSIONS}

The results suggest that no matter whether sap of Nitraria is added or not, the $\mathrm{NaCl}$ tolerance of 3 strains was all in $0 \sim 4 \%$, but 3 strains can not survive at $5 \% \mathrm{NaCl}$; with being added sap of Nitraria, the colony diameter of NHglj1 is significantly lower than that of control group $(P<0.05)$, and is $18 \% \sim 41 \%$ of control, However, the colony diameter of NHglj2 and LMw107 are 1.02 1.45 times than those of control group.

No matter sap of Nitraria is added or not, 3 endogenous strains separated from different tissues can grow over in $\mathrm{pH}$ 6 13 under $\mathrm{N}$-free conditions, and has application potential of survival nitrogen fixation under alkaline stress. The colony diameter of NHglj1 is significantly lower than that of control group $(P<0.05)$, and that means alkaline-resistance of NHglj1 is decreased when plant sap of Nitraria is added to. However, the colony diameter of NHglj2 and LMw107 are much greater than those of control group over in $\mathrm{pH}$ 6 13. These results indicate that the sap of Nitraria can improve the adaptation for parts of endogenous $\mathrm{N}$-fixing microbes to acidic and alkaline environments. But hosts plant may also restrict some endogenous microbe growth too much such as NHglj1 under alkaline conditions, but the reasons and mechanisms are not clear.

\section{ACKNOWLEDGMENT}

This work was financially supported by National Natural Science Foundation of China (NSFC) (31300389), The Natural Science Foundation of Guizhou Province (J[2014]2137) and the Ph.D. research project of Guizhou Normal College (13BS019).

\section{REFERENCES}

[1] W. E. Newton, "Symposium on Nitrogen Fixation." Bioscience, vol. 27, pp. 281-282, 1977.

[2] S. D. Fiore and M. D. Gallo, "Endophytic bacteria: their possible role in the host plant”. Nato Asi, Berlin: Springer-Verlag , 1995, pp. 169-187.

[3] S. Urquiaga, K. H. S. Cruz, and R. M. Boddey. "Contribution of Nitrogen Fixation to Sugar Cane: Nitrogen-15 and NitrogenBalance Estimates." Soil Sci. Soc. Am. J., vol. 56, pp. 105-114, 1992.

[4] B. Ye, A. S. And, and K. Minamisawa. "Effect of Inoculation with Anaerobic Nitrogen-Fixing Consortium on Salt Tolerance of Miscanthus sinensis," Soil Science and Plant Nutrition, vol. 51, Feb. 2005, pp. 243-249.

[5] R. Rodés, Z. Dong, F. G. Loiret, E. Ortega, D. Kleiner and P. Ortega-Rodés, "A putative new endophytic nitrogen-fixing bacterium Pantoea sp. from sugarcane," Journal of Applied Microbiology, vol. 97, March 2004, pp. 504-511.

[6] G. Zhang, Q. Mao, and H. E. Zhongyi, "Detection of Nitrogenase Activity and Phosphorus Dissolving Ability of Endophytic Isolates from Oryza rufipogon in Lingshui," Chinese Journal of Applied and Environmental Biology, vol. 12, April 2004, pp. 457-460.

[7] Z. X. Lv, J. D. Li and Z. Q. Zhu, "Inoculation, Isolation of endophytic diaztorophic bacteria and colonization in maize root tissue under gnotobiotic condition,” Chinese Journal of Applied and Environmental Biology, vol. 7, March 2001, pp. 207-212.

[8] R. K. Shrestha, and J. K. Ladha "Genotypic variation in promotion of rice dinitrogen fixation as determined by nitrogen-15 dilution," Soil Science Society of America Journal, vol. 60, June 1996, pp. 1815-1821.

[9] H. Ji, C. D. Pan, J. Zhou, M. Luo, W. X. Wang and D. Luo, "Adaptability of Endophytic Diazotrophic Bacteria Strains from Desert Shrubs to Diverse Environment Factors,” Journal of Desert Research, vol. 31, April 2011, pp. 942-947.

[10] P. Jha and A. Kumar, "Characterization of novel plant growth promoting endophytic bacterium Achromobacter xylosoxidans from wheat plant,” Microbial Ecology, vol. 58, Jan. 2009, pp. 179188.

[11] Y. X. Liu, "The Classification and Distribution of Genus Nitraria L. of China,” Research on Desert Control, No.7. Beijing: Science Press, 1965, pp. 76-83.

[12] S. M. Nie, L. Gao, Z. J. Yan, “ Study on vegetation characteristics and soil nutrition of Kubuqi Desert,” Pratacultural Science, vol. 27, August. 2010, pp. 23-28.

[13] D. F. Herridge, M. B. Peoples and M. B. Robert, "Global inputs of biological nitrogen fixation in agricultural systems,” Plant and Soil, vol. 311, 2008, pp. 1-18.

[14] S. Hara, Y. Hashidoko, R. V. Desyatkin, R. Hatano and S. Tahara, "High rate of N2 fixation by East Siberian cryophilic soil bacteria as determined by measuring acetylene reduction in nitrogen-poor medium solidified with gellan gum," Applied Environment Microbiology, vol. 75, Oct. 2009, pp. 2811-2819.

[15] S. Q. Zhang, Migration of Rhizobia inside Alfalfa Plants and Influencing Factors, PhD degree thesis: Gansu Agricultural University, July 2012, pp. 24-30. 